\title{
What do you mean by learning lab?
}

\section{Eric Sanchez ${ }^{1} \mathbb{C}$ - Elsa Paukovics ${ }^{1}$. Lilia Cheniti-Belcadhi ${ }^{2}$. Ghada El Khayat ${ }^{3}$. Bilal Said ${ }^{4}$. Ouajdi Korbaa ${ }^{2}$}

Received: 15 July 2021 / Accepted: 8 October 2021 /Published online: 26 October 2021

(c) The Author(s) 2021

\begin{abstract}
Digital and innovation competencies are nowadays highly required for students and faculty members in higher education institutions. We therefore need environments that incubate innovative learning scenarios to develop these competencies. We conducted a designbased research with the dual objective to develop learning labs in four universities and to document the design and implementation process and the addressed challenges. In this paper, we describe the concept of learning lab and the process of its setting up based on the experiments and observations conducted in the four learning labs in Lebanon, Egypt, Tunisia and Switzerland. A learning lab is defined as a physical, digital and human space for observation, experimentation and evaluation, to rethink and enrich learning and teaching attitudes and practices at the university. A learning lab is an incubator of pedagogical innovation and digital learning structured on three dimensions: spaces, activities and communities. We also identify the main challenges for the design and implementation of a learning lab. These challenges consist of the institutionnalisation of the learning lab and building, growing and nurturing a learning community.
\end{abstract}

Eric Sanchez

eric.sanchez@unige.ch

Elsa Paukovics

Elsa.Paukovics@unige.ch

Lilia Cheniti-Belcadhi

liliachenitibelcadhi@gmail.com

Ghada El Khayat

ghada.elkhayat@alexu.edu.eg

Bilal Said

bilal.said@gmail.com

Ouajdi Korbaa

Ouajdi.Korbaa@mars.rnu.tn

1 LIP/TECFA, University of Geneva, Geneva, Switzerland

2 ISITCom, University of Sousse, Sousse, Tunisia

3 Information Systems and Computers Department, Faculty of Commerce, Alexandria University, Alexandria, Egypt

4 LCPI, Arts, Sciences and Technology University in Lebanon (AUL), Beirut, Lebanon 
Keywords Learning Lab · Pedagogical innovation · Digital competencies · Design Based Research Methodology

\section{Introduction}

During the last few years, we have observed a huge need in educational systems to empower learners with competencies that are more and more needed in the labour market and for citizenship such as innovation, collaboration, problem solving, critical thinking and digital literacy. This leads academic institutions to work on teaching and learning approaches, to facilitate the development of such competencies and to promote pedagogical innovation and digital learning. Indeed, pedagogical Innovation, is now considered as a priority in many higher education institutions and almost systematically included into their strategies. The pandemic during the last months that resulted in an intensive use of technologies for learning and teaching has highlighted its value.

It is important to support the various academic actors in the process of integration of digital and pedagogical innovation. We therefore need support structures for educational innovation experimentation in universities. This support can be a physical and/or a digital environment to incubate pedagogical innovations and to facilitate the design and deployment of innovative and digital learning scenarios.

In this paper, we present the concept of learning lab, and analyse its specificity, characteristics and components. We also propose a model for the design, deployment and evaluation of learning labs in academic institutions. We detail the implementation and the experimentation of this model as well as the lessons learned in four academic institutions from four different countries.

This paper is structured as follows. In the first section, we introduce the general context of higher education, and more specifically aspects related to digitalisation and innovation. In the second section we present the concept of a learning lab as an environment dedicated to foster digitalisation and innovation. We describe its characteristics and give a summary of the research contributions related to this topic. In the third section, we first present our research questions and describe our research methodology related to these questions. We then propose in the fourth section our model to conceptualize a learning lab and describe the way this model has been implemented and deployed in a network of four academic partners as well as the lessons learned based on the data collected. The last section is dedicated to a conclusion and future research perspectives.

\section{Context: Digitalisation and innovation in higher education}

The ongoing sanitary crisis demonstrated that the digitalization of higher educational systems offers opportunities in terms of remote or blended learning and teaching. However numerous challenges still need to be faced and do not only rely on the technical dimension of learning and teaching. From the teachers perspective, there is a need to conceive and adopt more innovative pedagogy and the challenges 
rely on their scholarship of teaching and learning (SoTL) that should be driven by a systematic inquiry and involves critical reflection as well as scrutiny by peers (Kreber, 2002, Colet \& al., 2011). We know that the challenges consist of adopting a more constructivist approach (Biggs \& Tang, 2011), to improve students' assessment practices (Boud \& Falchikov, 2007) and to integrate ICT into their teaching practices (Cuban \& Jandrić, 2015). From the students perspective, there is a need to develop new ways of learning, for example, more autonomous and more collaborative learning methods, to develop students agency and digital skills (Trede al., 2012). From both perspectives, teachers and students need to develop digital competencies. These competencies are described in different general frameworks addressing citizens or more specific frameworks considering educators (see for example Redecker, 2017). The efforts made by higher education institutions for the development of these competencies aim to promote innovation in education.

Innovation is the enrichment of social practices (Chevallard, 1982) and is defined as a process which includes the production, acceptance and implementation of new ideas, processes, products or services (Marinova \& Phillimore, 2003). Babic and Nedelko (2020) consider innovation in higher education as a process which consists of institutional adaptation to changes in an ever changing environment. This adaptation enables higher education institutions to improve their existing practices. Innovation takes different forms and addresses different levels. O'Banion et al. (2011) emphasize the need to create a culture of innovation at the institutional level. The expression "culture of innovation" refers to a state of mind, shared values and practices that value imagination and creation, collaborative work, reflection about uses and users, favour interactions, sharing of knowledge, risk-taking, but also adaptation in iterations through mental and organizational flexibility (Forest, 2018).

Innovation in education is a challenge addressed by a consortium of four universities from Lebanon, Egypt, Tunisia and Switzerland (LETS) through the design of learning labs in four different countries and academic contexts. The consortium is involved in the design and the implementation of a learning lab dedicated to pedagogical innovation in each of the 4 universities. The LETS Learning Lab project's objectives consist of (1) fostering a culture of pedagogical innovation, (2) developing innovative educational practices and (3) developing the students and teachers digital skills. In the next section we present a brief review of the state of the art related to pedagogical innovation and present the definition that we propose for the learning lab concept.

\section{Learning lab, state of the art}

\subsection{Pedagogical innovation in higher education}

Pedagogical innovation relates to innovation in teaching and learning. Pedagogical innovation consists of changing the curricula and the way they are offered through the implementation of new methods for teaching and learning (Bajada et al., 2019). When innovative educational practices are being researched, the focus is often on digital technology. However, pedagogical innovation focuses on education and not on technology (Lison et al., 2014). Furthermore, innovation is context-dependent (Lison et al., 2014), which means that it is not the artifact or its features which is important but 
its introduction in a specific context. This implies that any attempt to innovate should be based on a context-analysis. Cros (2004) describes 5 components of pedagogical innovation: "the novelty, the object, the change, the finalized action and the process". In other words, pedagogical innovation is based on a contextualized and relative novelty and the change results from a finalized action, which is considered as part of a process.

In the last decade, pedagogical innovation has been widely explored with respect to school contexts but less commonly in higher education (Fraser, 2019). Thus, pedagogical innovation is still an important challenge for higher education institutions. Indeed, with the development of digital technology and the transformation of professions, teaching and learning methods need to evolve. In addition, the changing needs of students and teachers (Mai Walder, 2014) lead to the diversification of training modalities. However, pedagogical innovation is not limited to teachers' practices since the innovation in students' learning processes may be independent of any teaching practice (Silver et al., 1997). This means that students matter, and that pedagogical innovation should consider both teaching and learning practices.

The consortium of the LETS Learning Lab project addresses the issue of pedagogical innovation through the design and the implementation of a learning labs in each of their 4 universities.

\subsection{Learning lab, towards a definition}

Our approach to pedagogical innovation consists of the design and implementation of a learning lab in each university of the consortium. The term laboratory (lab) refers to both: a place dedicated to work (labor in Latin), to manufacture something and a place dedicated to scientific experimentation. Thus, the expression "learning lab" refers to both practice (to create something) and research (to experiment something) about learning. Within this context, learning can be understood in its broad sense as developing new teaching practices for teachers or new learning strategies for students. Therefore, a learning lab comes within the Scholarship of Teaching and Learning (Kreber, 2002).

We found a very limited number of research papers attempting to define what a learning lab is. Most of the other available resources are websites, brochures and blogs produced by stakeholders and practitioners. As a result, different definitions of what a learning lab is in higher education come from the hosting institutions websites. The Stanford Learning Lab ${ }^{1}$ created in 1997 is a place dedicated to carry out "projects to improve the quality of teaching and learning in higher education through effective application of information technologies and the sciences of learning". The EM Learning $L a b^{2}$ website states that "a learning lab is a place and an ecosystem for experimentation and innovation on new forms of collaborative work and collaborative learning. These innovative collaborative spaces make simultaneous use of digital tools, environments, equipment, learning materials and pedagogical methods that promote collective intelligence". The website of the Learning Lab Network ${ }^{3}$ provides criteria, actions and issues related to the learning labs.

\footnotetext{
1 http://sll.stanford.edu/

2 https://executive.em-lyon.com

3 https://www.learninglab-network.com/
} 
A literature review shows that there is a diversity of approaches followed by researchers. Thus, a learning lab is either a method of intervention, a program (Bal et al., 2018), a physical (Carron et al., 2018) or digital (Zinger et al., 2017) space, often located in the library of the university (Cartier, 2014). In terms of objectives, we found the same diversity: a learning lab is dedicated to address educational or social challenges such as racism and equity (Bal et al., 2018) and natural hazards (Shabudin et al., 2017). Some researchers consider that a learning lab aims at training students in general or improving the students' (Brehm \& Guenzel, 2018; Kift, 2017) or teachers' (Zinger et al., 2017) digital skills. We also found a diversity of contexts for the implementation of a learning lab such as governmental organizations, high schools (Bal et al., 2018), engineering schools (Carron et al., 2018) and universities (Kift, 2017).

Based on this literature review and from our perspective, we consider that a learning lab should enable a process aiming at both improving teaching and learning practices based on the use of digital technology in higher education (pedagogical innovation) and promoting a culture of innovation.

\section{Research questions and objectives}

Most definitions emphasize that a learning lab is dedicated to educational innovation and is based on the collaborative work of stakeholders. However, there is not yet a common vision about what a learning lab is. As a result, there is a need to conceptualize it and to understand how a learning lab can be developed and implemented to meet the challenges faced by the institutions involved in the LETS Learning Lab project.

This contribution aims to address 2 research questions (RQ):

- RQ1 relates to the conceptualization of learning labs to support pedagogical innovation in higher education. What are the core elements of a learning lab dedicated to pedagogical innovation and how these elements interact ? We address this question by building a model of a learning lab based on the empirical work carried out by the LETS Learning Lab Network. This model should emphasize the elements enabling to foster pedagogical innovation and to promote a culture of innovation.

- RQ2 relates to the description of the implementation of the proposed model in the 4 contexts addressed by the LETS Learning Lab Network. We consider the success of this implementation in different contexts as a proof of concept which values the model and validates its generecity. This success is assessed with data collected from the different contexts. We also want to describe the concrete elements that are produced in the different contexts as well as the difficulties faced, and the lessons learned.

In the next section we present in detail our research methodology and the research process that we have adopted to design and set up the learning labs. 


\section{Research methodology}

\subsection{A design-based research methodology}

The LETS Learning Lab project consists of combining theory and practice. Hence, the methodology is design-based. Design-Based Research (DBR) (Anderson \& Shattuck, 2012; Design-Based Research Collective, 2003; Penuel et al., 2007; Sanchez et al., 2017) is a specific type of collaborative research aiming at combining pragmatic and theoretical issues. DBR first perspective is focused on the design of a specific educational setting which is, for this project, a learning lab implemented in each of the 4 partner institutions. DBR stands out as a kind of engineering science, whose main aim is the intelligent transformation of practices. The second perspective is tightly related to the first one. DBR proposes some middle-range theories where the conceptual tools that are developed aim to improve practices.

We consider DBR to be particularly well-suited to our objectives. Indeed, the objectives of the LETS Learning Lab consortium are pragmatic. The project is driven by the willingness of 4 academic institutions to foster pedagogical innovation and to develop the needed instruments. In addition, through the development of a generic model of what a learning lab is, the consortium also aims to address theoretical objectives.

The achievement of the articulation of pragmatic and theoretical objectives is permitted by the core characteristics of DBR. First, the collaboration of the different stakeholders allows them to develop a solution adapted to their needs. The collaboration also allows the sharing and development of knowledge. Educational researchers, professors and lecturers, members of the university board and students participated in workshops dedicated to the design of the learning labs.

Second, DBR is iterative. Iterative cycles concern different stages of the DBR process (Kennedy-Clark, 2015): (1) The requirement and context analysis phase was conducted as a preliminary research (2)The development and implementation phase led to the development of different kinds of learning lab activities that are adapted to the needs and expectations of the stakeholders (3) these activities were tested and revised and a preliminary conceptual model was revised accordingly to the outcomes of the tests. Iterations enabled the improvement of the solution and the refinement of knowledge.

Third, DBR is contributive. The contribution of the project is both pragmatic (the physical and digital spaces designed and deployed for implementation of activities and theoretical (the proposed conceptual model of a learning lab).

Lastly, DBR is carried out in authentic contexts. The partner institutions constitute 4 specific contexts for the design and the implementation of 4 different learning labs. The comparison of the different learning labs enables us to distinguish what is generic and what is context specific regarding the proposed model.

\subsection{Narrative design}

In the following, we turn the implicit knowledge we used to model the learning lab into an explicit design narrative (Hoadley, 2004) through the description of the 


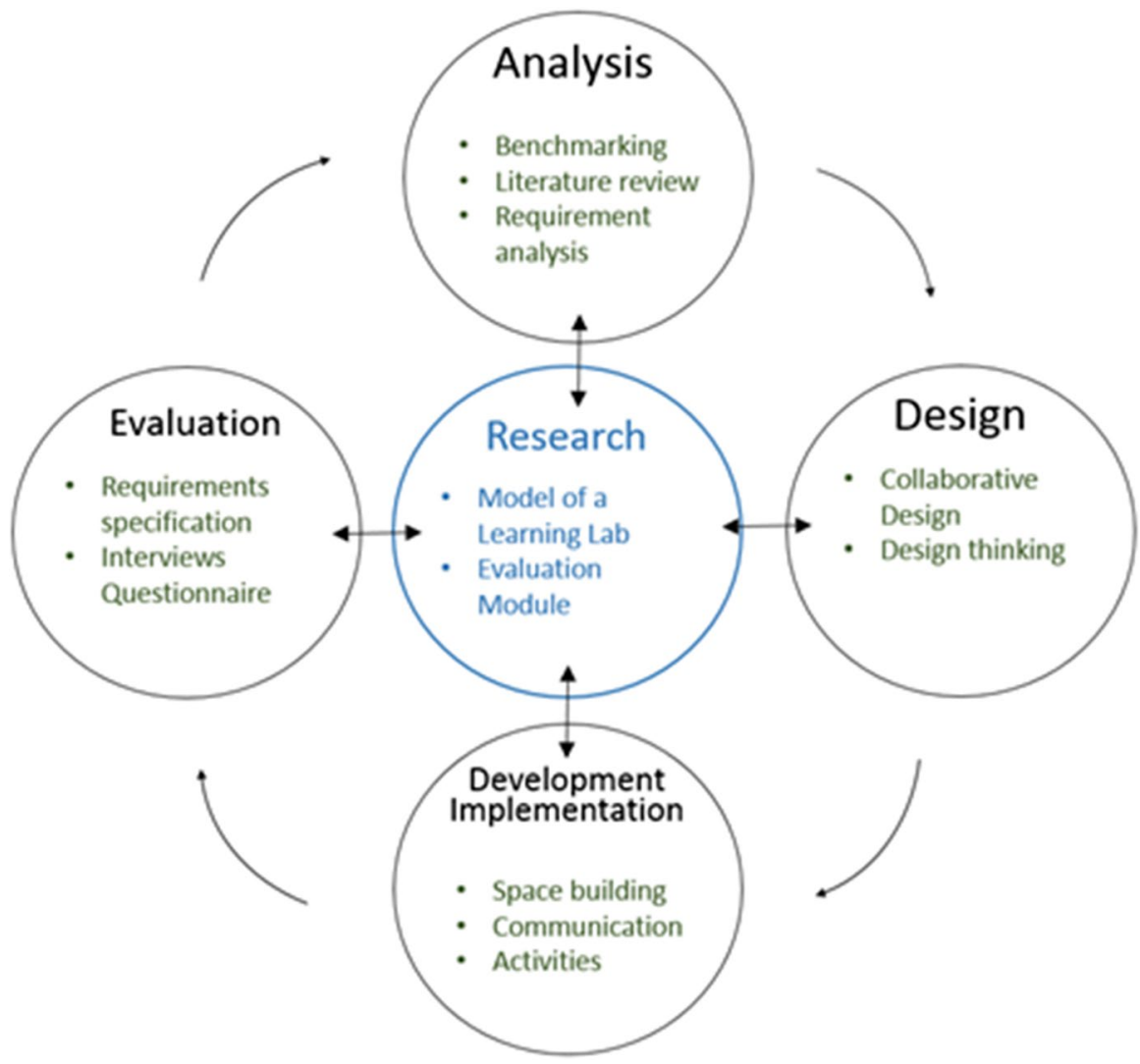

Fig. 1 Narrative Design of the Research Project

history and evolution of the design over time. Figure 1 illustrates the DBR process as a cycle of analysis, design, implementation and evaluation. This process is inspired by the ADDIE model for educational training (Branch, 2009).

Each stage of the process enables the production of knowledge (mentioned as the research stage) as shown in Fig. 1.

- Analysis: we performed benchmarking against the already existing learning labs, a literature review and a requirement analysis conducted through interviews with stakeholders. We also defined the problem in terms of audience, objectives and strategy.

- Design: two workshops were organized. The partners of the LETS Learning Lab project participated in a full day workshop dedicated to the design of the learning labs based on the outcomes of the previous analysis. Education researchers, professors and lecturers, members of the university board and students participated in a workshop dedicated to collaborative design (Kleinsmann, 2006). Based on an elementary model of the learning lab, the participants proposed some pre- 
liminary concrete ideas in terms of space, activities and community. The design provided the tasks and strategies of the network.

- Development and Implementation: Based on the assignment of roles and responsibilities, the different partners concretized the preliminary ideas into the design and the setting up of physical and digital spaces. We designed a communication plan and we organized face-to-face or online activities with teachers and students. These actions were implemented and monitored individually by each partner or collaboratively.

- Evaluation: We wrote the requirements specifications considering the generic and specific dimensions depending on the different partner institutions. In addition, we sent a questionnaire to the teachers and students who participated in the activities and interviews carried out with partners and participants. The evaluation process was conceptualized as an evaluation module, a fully-fledged component of the learning lab dedicated to make visible the weak points of our approach and the needed revisions of the design.

- Research: The information and the knowledge that emerged from the different steps of the DBR cycle enabled us to set up a model of the learning lab. The model was the result of (1) a literature review and a state of the art of the already existing learning labs, (2) the ideas that emerged during the design phases, (3) the lessons learned from the development and implementation phase and (4) the feedback from users since we paid specific attention to the module dedicated to activities monitoring (evaluation module).

This approach demonstrates that cooperative endeavours between stakeholders and researchers allows building fundamental research. Indeed, according to a practice-based evidence paradigm, researchers and stakeholders share the same ends that are both improving an instrument (i.e.a learning lab), and a better understanding of the fundamental features of this instrument. The evaluation of the learning lab model is based on the development and implementation of a proof of concept as a demonstration of the value of the theoretical model. As a result, we used the following criteria: (1) Setting up of learning lab spaces in the 4 partner universities, (2) Getting communities on board and involved in the proposed activities and (3) the design of activities.

This research methodology enabled us to design a model for a learning lab described in the following section.

\subsection{Data collected}

We also collected data from the different universities. Indeed, we developed a module as an integrated part of a learning lab. This module is dedicated to assess the acceptability, usability and usefulness of the activities implemented in the 4 universities. More precisely, usefulness refers to the extent to which the learning lab enables the project to meet the objectives (innovation and teachers professional development). Usability is addressed through the satisfaction of the users: Do they consider the digital and physical spaces adapted to the implémented activities? Do 
they consider that the proposed activities are suitable. Acceptability is addressed through the capacity of the learning lab to comply with the motivation and intentions of the users.

The module is mainly based on a questionnaire filled by the participants before and after each activity and semi-structured interviews with selected participants (Paukovics \& al., Submitted).

\section{The learning lab model}

One of our research objectives is to establish a model for a learning lab that can be used as a formal framework for design of this pedagogical innovation framework. A model is a simplified representation identifying interactions between various elements. We may identify two main categories "descriptive models" and "prescriptive models". Descriptive models describe an existing situation, whereas the second type of models represent an ideal solution for a given context. Initially, our approach was qualified as descriptive since the research work is based on our involvement in setting up a learning lab network. This model can also be considered prescriptive as we established a theoretical framework which offers the main requirements and specifications to be considered when setting up a learning lab.

In answering RQ1 (i.e.the conceptualization of what is a learning lab dedicated to pedagogical innovation and the modelling of its core elements), we separate the learning lab objectives, the evaluation module and the learning lab itself.

\subsection{The learning lab objectives}

The work done by the partners made visible that a learning lab should enable meeting 3 main objectives. These general objectives can be broken down into several specific learning objectives. Each activity conducted in a learning lab targets specific learning objectives. These objectives vary according to the universities. These learning objectives do not relate to disciplinary competences. The activities mainly target the development of soft-skills and digital skills, support reflexivity and foster the evolution of attitudes towards teaching and learning. More specifically, they target the use of educational technology and the development of a practitioner into a researcher posture (Elliott, 1990). A competency framework describes the competencies that teachers or students are expected to master. This framework is based on the European Framework for the Digital Competence (Redecker, 2017).

Besides the learning objectives, the learning lab aims to foster interaction between academic bodies from different departments/faculties in order to enable knowledge sharing and practices and the collaboration for the co-design and co-production of educational resources. The learning lab should also highlight the commitment of the academic bodies in pedagogical innovation,projects of production of resources through internal and external communication and the dissemination of resources. 
Finally, a learning lab is expected to create a favorable environment for pedagogical innovation based on the design, implementation and experimentation of innovative practices and digital technology.

\subsection{A 3-dimensional model}

Based on the literature review and on the collaborative design methodology, 3 core dimensions of the model emerged:

- The first dimension is constituted by the community. A learning lab is mainly defined by a community of teachers and students from a given university. By community, we mean a flexible organization oriented towards shared goals, a collective experience and a "shared microculture" based on values, practices, conversational rules and behaviors (Preece \& Maloney-Krichmar, 2003). The community is not limited to a learning community (Dionne et al., 2010). We managed to identify different other stakeholders who belong to this community and should be considered. These stakeholders are from the university (technical or administrative staff, pedagogical engineers and specialists of educational technology) and external (different persons who are interested in participating and experts who are invited to give talks or organise workshops). Thus, a first challenge for the design and implementation of the learning lab consists of building, growing and nurturing this community.

- We argue that a learning lab is also defined by activities, which constitute the second dimension. These activities are concrete learning scenarios designed, organized or facilitated by the learning lab. They require participation and commitment of the community in order to meet participants' learning objectives as well as one or more of the learning labs' objectives. Thus, in order to meet the objectives and values, these activities must allow the discovery, observation, experimentation, and evaluation of teaching and learning practices. Activities should also include innovative modalities, be learner-centered, encourage sharing of knowledge, be assessed and formalized, be made visible and valued at a university level.

- A learning lab is also defined by hybrid spaces composed of physical and digital spaces, which define the third dimension. The physical space encompasses different rooms designed to accommodate the activities and the community they aim to host. The digital space takes the form of a digital platform allowing communication (webinars, meetings) and storing and dissemination of pedagogical resources. The digital space also encompasses social networks that enable the building of the community. The main characteristics of the learning lab spaces are their affordance and modularity so that they can (1) adapt activities to users needs, (2) promote collaboration and sharing between heterogeneous audience, (3) evolve according to users needs of users and technological advances, (4) make visible the pedagogical innovations undertaken by members of the community. 


\section{Community}

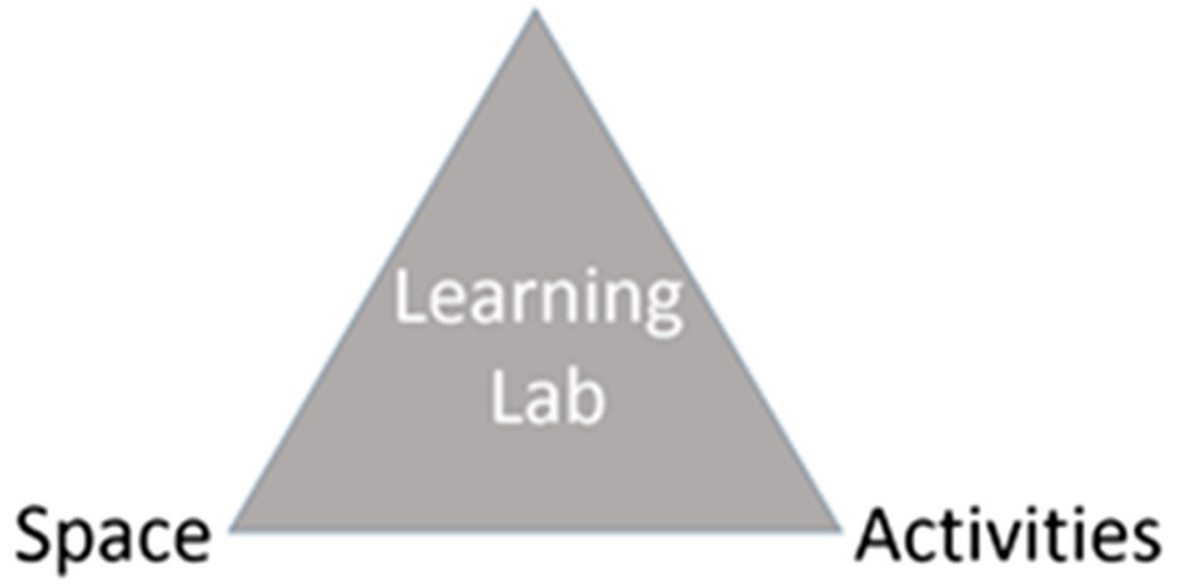

Fig. 2 The learning lab as a 3-dimensions model

The 3 dimensions are not isolated. They interact so that each dimension is impacted by the choice made for the design and implementation of the two others dimensions. This leads to the fact that each decision taken in terms of design and implementation should be based on a systemic approach.

Based on the reflection that emerged from the collaborative design workshops and the concrete implementation of a learning lab in each partner's university, the members of the network agreed on a common definition about what a learning lab is. This definition states that a learning lab is a physical, digital and human space for observation, experimentation and evaluation, to rethink and enrich learning and teaching attitudes and practices at the university (Fig. 2).

\subsection{The evaluation module}

The evaluation of the learning lab is an integral part of its implementation. Thus, we designed an evaluation module based on the evaluation model of Tricot et al. (2003) addressing usefulness, usability and acceptability. Thus, the evaluation module includes different tools for the articulation of the purpose of the evaluation (the decisions expected to be taken) and what is to be evaluated (objects to be evaluated). The evaluation module aims to link the meaning of the information (the referent), the representative aspects of the object to be evaluated on which a judgement must be made (criteria) and the clues, traces and observable characteristics for judging the degree of attainment of the criterion (indicators).

The tools of the evaluation module allow the monitoring of the learning lab. The different elements of each activity are recorded and documented in an activity plan. Online questionnaires are filled in by participants. Registration forms record the characteristics and the objectives of the participants while feedback forms record their opinions about the activities. Interviews that are carried out with selected 
participants give an in-depth knowledge of the usefulness, usability and acceptability of the learning lab.

In the next section, we present the implementation and deployment of this model in the learning lab network of the four universities.

\section{Implementation of the learning labs}

In the following, we show the way each partner, from the 4 different universities, is implementing the generic model presented above, faced difficulties and lessons learned (RQ2). This implementation is described according to the three dimensions of our learning lab model.

\subsection{Space}

The physical space can be illustrated by the space designed by the Swiss University. The results of the collaborative design thinking workshop dedicated to conceptualizing this space consisted in 3 different concepts. Different common elements and specific ideas were selected from these concepts. The following description results from this selection. The physical space of the learning lab is composed of a Teaching \& Learning space, a room dedicated to experiment and record innovative teaching practices. The Living Space is another room where meetings, seminars, lectures and social interactions can take place. The Living Space is also a hub to the other rooms, and it is open and visible to potential visitors who circulate in the building. The Project Space is a room where people can find all the necessary amenities to initiate and carry out a project of pedagogical innovation. The project space hosts a Fab Lab or makerspace, i.e.useful equipment for prototyping (2D printer, laser cutting...). This physical space is a modular space so that it can (1) adapt to the activities of users, (2) promote collaboration and sharing between heterogeneous audiences, (3) evolve according to the needs of the university and technological advances and (4) encourage the appropriation of the space by users.

The digital space from the Tunisian University consists of a Facebook group that hosts approximately 1200 members. The Facebook group hosts pedagogical resources and is used for advertising the learning lab activities and for communication between members. The digital space also comprises a digital platform (Microsoft Teams) for hosting webinars and web-conferences.

In Alexandria University, the physical space is composed of different areas designated for specific purposes. The Pedagogical Innovation Welcome Hall showcases the achievements of the learning lab and publicizes its activities. The Pedagogical Innovation Incubator incubates practices and tools that support and improve education. A Green Screen Video Production Studio as well as an Audio Production Studio are used to produce content. The team working stations are within this space which also includes a place for experimentation and a Training Room. Digital spaces complement this physical space and allow sharing and meeting. 
Whether it is for the physical space or digital space, the key concept is modularity. Indeed, the spaces serve a variety of activities adapted to the diversity of stakeholders. As a result, the spaces should be designed so that it would be possible to adapt them to various users needs. For example, in the Swiss University, in terms of physical space, the modularity is based on movable partitions of the rooms and furniture that can be easily arranged according to user needs. In terms of digital space, it consists of different tools such as learning management systems, blogs, video conference platforms and social networks.

\subsection{Activities and actions}

The learning lab activities are based on innovative training modalities. They are defined and designed according to the following information: (1) the description includes the name and the topic of the activity (e.g. game-based learning), the type of activity (e.g. workshop, webinar...), the place, the duration and the date on which the activity is held. The activities are also defined by (2) the objectives of the activity. The learning objectives are inspired by the European Framework for the Digital Competence [2]. An activity also encompasses (3) the actors and tasks that will be performed. This category is a description of the activity in terms of learning design, organization, people involved and target audience. (4) Resources consist of all the tools and documents useful to perform the activity (e.g.registration form, communication tools) or produced by the activity. (5) Assessment is also an important dimension of the activity. Specific feedback questionnaires or interviews with people who were involved in the activity allow us to know if the objectives have been achieved. Each activity is documented by specific comments from the organizers and from the results of the evaluation of the activity. The traceability of the activities appeared important to us.

The "game-based learning workshop" that took place in the Lebanese University is an example of such activity. The activity consisted in a 1-day workshop dedicated to a short lecture about the principles of game-based learning. The participants also participated in a role-play game about the relationship between teachers and students (the Knives and Glasses role-play game). They discussed what game-based learning means during a debriefing. They also collaboratively designed a gamebased learning sequence adapted to their teaching practices. Each participant was expected to understand the principles of game-based learning and to be able to apply these principles in one's own practice. Two experts in game-based learning designed and led the workshop. The participants were voluntary teachers and staff from the University. The resources used for the activity were the game material and a roadmap for game design. The participants produced and discussed 3 game prototypes. They filled out a feedback questionnaire, and the organizers took notes during the workshop.

The consortium tested different formats of activities such as "Lunch \& Discover" (a 45 min meeting dedicated to discuss a specific topic or to discover a learning/ teaching tool under the supervision of an expert during lunch time), "Breaking Infox", an hour discussion with a leading expert to address a "hot" learning or 
teaching issue (e.g.students online assessment), a roundtable about a specific topic considered important (e.g. a roundtable with students about their needs in terms of remote learning) and "E-learning Fake News", blog posts written by leading experts and dedicated to deconstruct misconceptions about teaching and learning. Other activities by the consortium included celebrating important events relating to education such as the International Day of Education on January 24th through the 100X100 OERWiki@MENA initiative having the objective to create 100 open educational resources in 100 days.

The main characteristic of the activities designed and deployed is that they target pedagogical innovation and engage a reflection of all involved participants on their learning and teaching practices. The design of an activity is time and resources consuming and the added value of the network lies in the possibility to share and exchange resources, experts and ideas.

\subsection{Community}

Depending on how the members of the learning lab community were involved, we define 3 categories of members. The spectators are members who are willing to be aware of what happens within the community and learn while observing when the activity takes place. The participants are active members who are deeply involved in the activities. Contributors are members involved in the organisation of the activity. They also produce educational resources. It is worth noting that not only academic experts can be contributors. For example, students can participate in a round table dedicated to sharing experiences about strategies for remote learning.

The building of the community follows a communication plan based on the use of social networks, disruptive communication and on the collaboration with the communication board of the university. In order to build the community and to ensure people's commitment, various means can be used such as newsletters, social networks, logo for corporate identity, creation of an online "learning lab member" profile, etc.... The measures to be taken must be consistent with the specific target audience, the learning lab objective, the activities and the human and financial resources available.

The communication plan of the Swiss University encompasses different specific measures adapted to the different academic bodies. Teachers are informed of the activities via a newsletter as well as the announcement of the different events via a mailing list. The social networks (Instagram and Facebook) and different disruptive communication measures are used to raise students' interest. For example, remote telepresence devices were used to meet them in the working rooms and the university restaurants.

In the Tunisian University, different initiatives took place to build the community. Several online activities of interest to both students and professors were organized. Different challenges and initiatives were launched in order to attract a community and to have it loyal to the learning lab. Pedagogical innovation competitions were introduced to help grow the community. They targeted both students and professors. Another factor that helped develop the community was the support provided by the 
learning lab network. Support groups and help desks were created and training was delivered to support professors in online teaching. Seasons of training were launched under specific themes or titles like "Ramadan Gana" Workshop Series, Do22o Eshamassi Workshop series, among others. This tightened the links between the community and the learning lab. Lots of communication channels were used to maintain contact with the community. They included both formal and informal means of communication.

\subsection{Acceptability, usability and usefulness of the activities}

Some figures show that the learning labs complies with the motivation of the users. At the Swiss and Tunisian universities, 10 to 30 participants were involved in each activity. In addition, the Facebook group of the learning lab from Sousse has now more than 1.200 members. Some resources produced by the Swiss Learning Lab have more than 20.000 readers. However, we found that most participants are mainly passive and more interested in getting information than sharing their teaching and learning experience. As a result, we paid specific attention to organizing activities fostering active participation. For example an online roundtable with students from different universities was dedicated to discuss the impact of the pandemie on students. The Swiss Learning Lab also organized an activity dedicated to the collaborative writing of 10 tips about online assessment.

The satisfaction of the users (usability) was assessed by a questionnaire. We got positive feedback regarding the time-slot dedicated to the events (mainly during lunch-time), the topics of the activities (but the feedback comes from people who participated and who are a priori interested) and the format of the activities (exchange between different kind of participants, quality of the exchanges...) but mixed feelings regarding the duration of the activities (too long or too short depending of the participants). We learnt that expectations and availability vary among participants and that there is a need to be clear about the objectives of the activities and to vary the type of activities.

Regarding the to which the learning lab enables to meet the objectives (innovation, teachers professional development, students agency), a direct and short time assessment is not feasible. That is why we asked the participants what they learnt, if they want to go deeper into the topic and if they plan to take into account this knowledge for their teaching and learning practices. We got positive feedback. All participants confirmed that that they learnt something and that it should impact their learning or teaching practices. In addition many participants expressed their willingness to enrich their teaching and learning practices. These observations confirm that the learning lab fosters a culture of innovation or, at least, manages to attract people who are already ready to innovate. However, we are not able to assess if this willingness led to concrete changes. We consider that the impact of a learning lab may vary depending on the participants profile and context and that it is difficult to assess this impact beyond the expression of the satisfaction of the participants. As a result, the impact of a learning lab is a long process and should be considered and measured over the long term. 


\section{Discussion and lessons learned}

The implementation of a learning lab dedicated to pedagogical innovation is a complex process. Table 1 summarizes the main issues faced by the consortium for the design, development, implementation and evaluations processes of the learning labs, details how these issues have been addressed and gives some examples from the LETS Learning Lab project.

A first issue lies in performing a design-based on theoretical and empirical knowledge. We adapted ADDIE, a pedagogical engineering framework (Branch, 2009) so that this framework allows designing specific spaces and activities, and also building a learning community. This framework also aims to foster the reflexivity of the stakeholders towards the taken decisions, based on the evaluation module.

The second issue consists of enabling a continuous assessment process for reflecting on the scientific issues related to various contexts. This issue has been addressed by developing an international network of researchers interested in educational innovation. The network offers the opportunity to reach the needed critical mass of experts and the diversity of needed expertises, and the possibility of learning labs deployment in various contexts.

A third issue is related to the involvement of stakeholders. This issue has been addressed in considering these stakeholders as co-designers for the whole process (analysis, design, implementation, evaluation). They have been invited to participate in a collaborative design workshops according to a bottom-up approach.

The institutionalization of the learning lab is a fourth and major issue. The support from the academic board and acceptance by the academic community depends on the alignment of institutional objectives with learning lab objectives and components. It should be profiled as an institutional project. For example, the activities that take place in the learning lab should not be competitive with the activities performed by the teacher training service of the university. In addition, the design of the activities needs to take into account the institutional objectives which are part of an overall strategy. The culture of innovation relies on the mutual definition of innovation (O'Banion et al., 2011). Therefore, it is important that the learning lab's objectives must be aligned with the institutional strategy of the university and supported by its board.

The issues listed above are considered as the main issues that we have observed in this research on the design and implementation of learning labs in the four universities.

\section{Conclusion and future work}

In this paper, we proposed a model for the design of learning labs, based on three components specifically community, activity and spaces. Following a design based research methodology, The proposed learning lab model emerged from a 


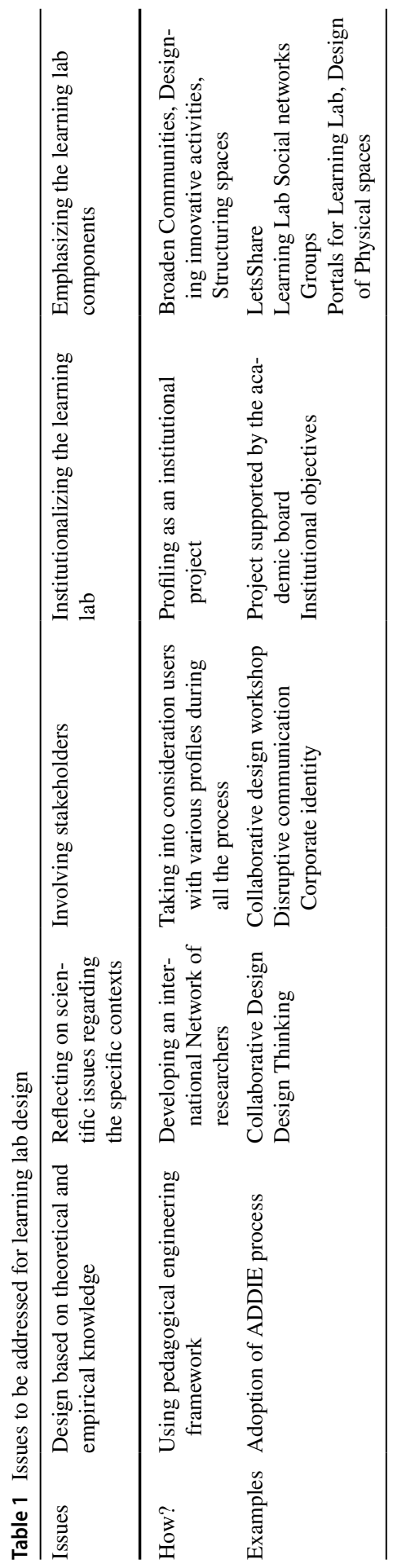


literature review, the collaborative design performed by a multidisciplinary team of researchers and stakeholder and an empirical and iterative process dedicated to test preliminary ideas. The successful deployment of our model in four universities and therefore in different cultural and academic contexts, enabled us to confirm its value and, to some extent, to show its genericity. This work confirms the relevance of the way of thinking about a learning lab in terms of spaces, community and activities.

The work carried out also demonstrates the need for a bottom-up process based on the needs and the participation of stakeholders and the building of a community. Another lesson learned is that the institutionalization of the learning lab is a key to a successful implementation. The work also demonstrates the difficulty to assess the impacts of a learning lab due to its long-term effect on the improvement of teaching and learning practices and the development of digital skills.

The currently ongoing work of the consortium is focusing on ethics, data privacy and the business models for the learning labs implemented by the different partners of the network. Further work should be done by the LETS Learning Lab Network, to broaden its community, and to further develop its activities and to better structure the digital and physical spaces.

Acknowledgements the authors thank the State Secretariat for Education Research and Innovation (SEFRI) and the University for Applied Sciences and Arts Western Switzerland (HES-SO), Leading House MENA, for their financial support.

Funding Open Access funding provided by Université de Genève.

Data Availability Non available.

Open Access This article is licensed under a Creative Commons Attribution 4.0 International License, which permits use, sharing, adaptation, distribution and reproduction in any medium or format, as long as you give appropriate credit to the original author(s) and the source, provide a link to the Creative Commons licence, and indicate if changes were made. The images or other third party material in this article are included in the article's Creative Commons licence, unless indicated otherwise in a credit line to the material. If material is not included in the article's Creative Commons licence and your intended use is not permitted by statutory regulation or exceeds the permitted use, you will need to obtain permission directly from the copyright holder. To view a copy of this licence, visit http://creativecommons.org/licen ses/by/4.0/.

\section{References}

Anderson, T., \& Shattuck, J. (2012). Design-based research: A decade of progress in education research? Educational Researcher, 41(1), 16-25.

Babic, V., \& Nedelko, Z. (Eds.). (2020). Handbook of research on enhancing innovation in higher education institutions. Information Science Reference.

Bajada, C., Kandlbinder, P., \& Trayler, R. (2019). A general framework for cultivating innovations in higher education curriculum. Higher Education Research \& Development, 38(3), 465478.

Bal, A., Afacan, K., \& Cakir, H. (2018). Culturally Responsive school discipline : Implementing learning lab at a high school for systemic transformation. American Educational Research Journal, 55(5), 10071050 . 
Biggs, J. B., \& Tang, C. (2011). Teaching for quality learning at University (4th ed.). Society for Research into Higher Education \& Open University Press.

Boud, D., \& Falchikov, N. (Eds.). (2007). Rethinking assessment in higher education. Routledge.

Branch, R. (2009). Instructional design: The ADDIE approach (Vol. 722). Springer Science \& Business Media.

Brehm, L., \& Guenzel, H. (2018). Learning Lab "Digital Technologies" - Concept, Streams and Experiences 4th International Conference on Higher Education Advances (HEAd'18). Universitat Politecnica de Valencia

Carron, T., Houzet, G., Abed, H., Pernelle, P., Lainé, P., \& Talbot, S. (2018). Teaching digital literacy: The outcomes from a learning lab. Journal of Electrical Engineering, 6(2), 75-84.

Cartier, L. (2014). The flexible learning lab. Knowledge Quest, 42(4), 1094-9046.

Chevallard, Y. (1982, 5 au 17 juillet 1982). Sur L'ingénierie Didactique, Texte préparé pour la 2e école d'été de didactique des mathématiques, Orléans

Cros, F. (2004). L'innovation scolaire aux risques de son évaluation. L'Harmattan.

Cuban, L., \& Jandrić, P. (2015). The dubious promise of educational technologies: Historical patterns and future challenges. E-learning and Digital Media, 12(3), 425-439. SAGE Journals.

Design-Based Research Collective. (2003). Design-based research: An emerging paradigm for educational inquiry. Educational Researcher, 32(1), 5-8.

Dionne, L., Lemyre, F., \& Savoie-Zajc, L. (2010). Vers une définition englobante de la communauté d'apprentissage (CA) comme dispositif de développement professionne. Revue Des Sciences de L'éducation, 36(1), 2543.

Elliott, J. (1990). Teachers as researchers: Implications for supervision and for teacher education. Teaching and Teacher Education, 6(1), 1-26.

Forest, J. (2018). Créer une culture de l'innovation. La Recherche (536).

Fraser, S. (2019). Understanding innovative teaching practice in higher education: A framework for reflection. Higher Education Research and Development, 38(7), 1371-1385.

Hoadley, C. (2004). Methodological Alignment in Design-Based Research. Educational Psychologist, 39(4), 203-212. https://doi.org/10.1207/s15326985ep3904_2

Kennedy-Clark, S. (2015). Research by design: Design-based research and the higher degree research student. Journal of Learning Design, 8(3), 108-122.

Kift, K. (2017). What Did the Disruptive Media Learning Lab Ever Do for Us? Insights, 30(3), 11-19.

Kleinsmann, M. (2006). Understanding collaborative design. Technische Universiteit.

Kreber, C. (2002). Teaching excellence, teaching expertise, and the scholarship of teaching. Innovative Higher Education, 27(1), 5-23.

Lison, C., Bédard, D., Beaucher, C., \& Trudelle, D. (2014). De l'innovation à un modèle de dynamique innovationnelle en enseignement supérieur. Revue internationale de pédagogie de l'enseignement supérieur 30(1).

Mai Walder, A. (2014). The concept of pedagogical innovation in higher education. Education Journal, 3, 195-202.

Marinova, D., \& Phillimore, J. (2003). Models of innovation. In V. Shavinina (Ed.), The International handbook on innovation (pp. 44-53). Elsevier.

O'Banion, T., Weidner, L., \& Wilson, C. (2011). Creating a culture of innovation in the community college. Community College Journal of Research and Practice, 35(6), 470-483.

Penuel, W., Roschelle, J., \& Shechtman, N. (2007). The WHIRL co-design process: Participant experiences. Research and Practice in Technology Enhanced Learning, 2(1), 51-74. https://doi.org/10. 1142/S1793206807000300

Preece, J., \& Maloney-Krichmar, D. (2003). Online communities. In J. Jacko \& A. Sears (Eds.), Handbook of human-computer interaction. Lawrence Erlbaum Associate Inc.

Redecker, C. (2017). European Framework for the Digital Competence of Educators: DigCompEdu. In Y. Punie (Ed.), EUR 28775 EN. Publications Office of the European Union,.

Sanchez, E., Monod-Ansaldi, R., Vincent, C. , et al. (2017). A praxeological perspective for the design and implementation of a digital role-play game. Educ Inf Technol 22, 2805-2824 . https://doi.org/ 10.1007/s10639-017-9624-Z

Shabudin, A., Azhar, S., \& Foo Ng, T. (2017). Learning lab on disaster risk management for sustainable development (DRM-SD): An evaluation. International Journal of Climate Change Strategies and Management, 9(5), 600-625.

Silver, H., Hannan, A., \& English, S. (1997). Innovation: questions of boundary. Working Paper No.2. 
Trede, F., Macklin, R., \& Bridges, D. (2012). Professional identity development: A review of the higher education literature. Studies in Higher Education, 37(3), 365-384. https://doi.org/10.1080/03075 079.2010.521237

Tricot, A., Plégat-Soutjis, F., \& Camps, J.-F. (2003). Utilité, utilisabilité, acceptabilité : interpréter les relations entre trois dimensions de l'évaluation des EIAH. In C. Desmoulins, P. Marquet, \& D. Bouhineau (Eds.), Actes de la conférence EIAH 2003 (pp. 391-402). ATIEF \& INRP.

Zinger, D., Naranjo, A., Amador, I., Gilbertson, N., \& Warschauer, M. (2017). A design-based research approach to improving professional development and teacher knowledge: The case of the Smithsonian Learning Lab. Contemporary Issues in Technology and Teacher Education, 17(3), 388410.

Publisher's note Springer Nature remains neutral with regard to jurisdictional claims in published maps and institutional affiliations. 